\title{
Dose verification of intensity modulated radiotherapy in head and neck tumors
}

\author{
Rasha A Elawady ${ }^{1}$, Ehab M Attalla1, ${ }^{1}$, Wael M Elshemey ${ }^{3}$, Tarek Shouman ${ }^{1}$, Anwar A Alsayed ${ }^{3}$ \\ ${ }^{I}$ Department of Radiotherapy \& Nuclear Medicine, National Cancer Institute, Cairo University, Cairo, Egypt. \\ ${ }^{2}$ Physics Unit-Radiation Oncology, Children's Cancer Hospital, Cairo, Egypt. \\ ${ }^{3}$ Department of Biophysics, Faculty of Science, Cairo University, Cairo, Egypt.
}

Received January 19, 2014; Revised April 12, 2014; Accepted April 20, 2014; Published Online May 04, 2014

\section{Technical Report}

\begin{abstract}
Purpose: To evaluate the agreement between measured and calculated doses for head and neck tumors using different gamma criteria and to establish quality assurance protocol for the delivery of IMRT in The National Cancer Institute in Cairo. Methods: The dose is calculated for 30 patients using CMS Treatment Planning System. The ionization chamber $\left(0.6 \mathrm{~cm}^{3}\right.$ Farmer type is used for point dose measurements. The 2D-array (PTW 729) and GafChromic films (EBT2) are used for 2D graphical dose distribution. Four different gamma criteria of dose difference (DD) and distance to agreement (DTA) (3\%/3 mm, 3\%/5 mm, 4\%/4 $\mathrm{mm}$ and 5\%/5 mm DD / DTA) are selected. These criteria are evaluated while suppressing the dose of 10\%, 20\% or 30\% from dose distribution. Results: Point dose evaluations using the ion chamber ranged from $-2.6 \%$ to $3.7 \%$ (mean and standard deviation of $0.46 \pm 1.7)$. Significant differences are observed between the films and $2 \mathrm{D}$-array for all criteria except the $3 \% / 5 \mathrm{~mm}$ criteria $(96.89 \pm 2.2 \%$ vs. $94.81 \pm 4.2 \%(\mathrm{p}<0.01))$. Conclusion: Differences may exceed about $3 \%$ when the ionization chamber is present in steep dose gradient regions. The present results suggest the gamma criteria of $3 \% / 5 \mathrm{~mm}$ as the most suitable criteria for IMRT quality assurance. This gamma criterion of $3 \% / 5 \mathrm{~mm}$ favorably exceeds $95 \%$ in case of maximum dose while suppressing the dose of $20 \%$.The use of 2D-array can reduce the IMRT QA workload.
\end{abstract}

Keywords: Quality Assurance; Intensity Modulation; Ionization Chamber; 2D-Array; GafChromic Films

\section{Introduction}

The standard quality assurance (QA) for IMRT before the patient treatment is to use an ionization chamber for absolute dose measurements and 2-dimensional (2D)-array or films for relative dose evaluation. ${ }^{1,2}$ During the introduction of intensity-modulated beams applied for IMRT, the physics community started to perform more extensive verification in 2D (planes) and even in 3D (volumes). Burman et al ${ }^{3}$ introduced a concept for IMRT verification. Kron et al ${ }^{4}$ performed a check of absolute dose calibration in a slab phantom. This study found that the dose delivered to the ICRU reference point was correct in all centers. They also found that the absolute dose calibration and the mean dose in the

Corresponding author: Rasha A Elawady; National Cancer Institute, Cairo University, Cairo, Egypt.

Cite this article as:

Elawady RA, Attalla EM, Elshemey WM, Shouman T, Alsayed AA. Dose verification of intensity modulated radiotherapy in head and neck tumors. Int J Cancer Ther Oncol 2014; 2(3):02037. DOI: 10.14319/ijcto.0203.7 target volume were $\pm 5 \%$ for all participating centers. Dong et al ${ }^{5}$ completed a dosimetric verification of 200 cases of head and neck using ionization chamber for patient-specific quality assurance. Most treatments were delivered using the step-and-shoot multileaf collimator with a 6-MV photon beam. The measured difference was greater than $3.5 \%$ for 14 cases. Low and Dempsey ${ }^{6}$ typically used 5\% and 2-3 mm as tolerance values of the $\gamma$-distribution during their clinical evaluation. In a more recent study Childress et $a l^{7}$ analyzed about 850 films resulting from IMRT plan verification. Their results showed no dependence on energy, accelerator or treatment site, but varied for the different QA phantoms and treatment planning systems applied in their study. Their preferred gamma index tolerance criteria were $5 \%$ and 3 $\mathrm{mm}$. Saminathan et al ${ }^{8}$ investigated the use of 2D-array compared to films in the verification of IMRT. Their measurements showed that the 2D-array could be used to quantifying absolute dose and could be also used for routine quality assurance checks like flatness, symmetry, field width, and penumbra of the linear accelerator beam. 
IMRT is an optimal technical approach for the treatment of head and neck tumors. This is because of the presence of anatomically critical structures close to the targeted cancer tissue. ${ }^{9}, 10,11$ This complex technique requires a highly efficient treatment-verification process. ${ }^{4}$ The IMRT delivery quality assurance mostly consists of delivering the IMRT plan to a phantom and then comparing the 2D dose distribution calculated by the treatment planning system (TPS) with

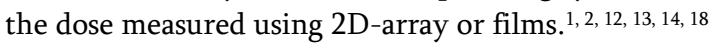

A "reasonable" choice of a specific combination of gamma evaluation and acceptance criteria should be based on the accuracy of the applied measurement procedure, its workload, and the ability to detect problem areas in the intended dose distribution. Combinations of gamma evaluation and acceptance criteria depend on many factors including the dosimetric equipment, calculation and measurement grid, and the data analysis software. It is therefore virtually impossible to provide general recommendations applicable for all situations. ${ }^{13}$ For these reasons, the goal of this work is studying the compatibility of IMRT dose calculations for head and neck tumors in TPS with dose measurement in Linac at the National Cancer Institute, Cairo, Egypt. Several tests are performed using ionization chamber, 2D array ionization chambers and films. Ionization chamber is used for point dose measurements. 2D array ionization chambers and films are used to display 2D graphics of dose measurements. This is to establish a protocol to present a complete QA process, evaluate the usefulness of the investigated methods and suggest the use of faster and more efficient dosimetric tools for the dose verification in IMRT technique in our institute.

\section{Methods and Materials}

\section{QA Tools}

The dose measurements are done in rectangular parallel slabs of water equivalent phantom. The phantom dimensions are $30 \times 30 \times 25 \mathrm{~cm}^{3}$. Three different tools are used in this study:

1. Ionization Chamber: $0.6 \mathrm{~cm}^{3}$ farmer type ion-chamber and unidose electrometer (PTW, Freiburg, Germany) are used for point dose measurements. ${ }^{12,13,14,15}$ A sandwich setup of water equivalent PTW RW3 plates with a stack of $15 \mathrm{~cm}$ below and $10 \mathrm{~cm}$ above the ionization chamber are used. The phantom arrangement is scanned in CT with slice thickness of $0.5 \mathrm{~cm}$. The scanned phantom is imported via DICOM to XIO treatment planning system.

2. 2D-array: PTW 2D-array 729 consisting of a plan matrix of $27 \times 27$ air-filled ionization chambers is used (PTW, Freiburg, Germany). The detector spacing (center to center) is $1 \mathrm{~cm}$. The dimensions of each detector are $0.5 \times 0.5 \times 0.5 \mathrm{~cm}^{3}$.
3. GafChromic films: EBT2 GafChromic films, each of dimensions $8 \times 10$ inches, are used (ISP-USA). The irradiated films are scanned with Vidar VXR-16 Dosimetry PRO scanner. MEPHYSTO mc2 software (PTW, Freiburg, Germany) is used for film analysis. Films are stored in light-tight envelopes under constant atmospheric conditions. The same time difference is maintained between the film irradiation and scanning. Edges of the films are not included in the region of interest to avoid artifacts.

2D-array and films are used for dose verification for treatment planning system. ${ }^{12,13}$ The same setup of water equivalent PTW RW3 plates used for point dose measurements is used for $2 \mathrm{D}$-array and film measurements. The phantom is irradiated using the same monitor units and same beam setup as the patient treatment setup with Elekta Precise linear accelerator (step-and-shoot IMRT delivery). 2D-array and films display 2D graphics and transfer the acquired data to the Verisoft software. Doses are calculated in cGy for 30 patients by inverse planning optimization using CMS XIO treatment planning system version (4.40). Seven beams are calculated for every plan. The number of segments may exceed about 120 segments for a head and neck plan. All of the measurements are performed on step and shoot Elekta Precise ${ }^{\circledR}$ linear accelerator.

\section{Verisoft software}

The Verisoft software assists physicists in comparing dose distributions in IMRT verification phantom with dose distributions computed by radiotherapy treatment planning system. Matrices of measured and calculated points of an IMRT beam are compared by subtracting the matrices and visualizing the results. The software supports the gamma evaluation method, it helps in locating hot and cold spots and determines maximum and average deviation between a calculated and measured plan.

In this study, the Verisoft verification software is used to compare gamma distribution for calculated dose (cGy) using TPS and measured dose using films and 2D-array. This is to find out what percent of pixels passing certain criteria imitate a good quality plan. Two tests are performed for the comparison between measured dose from Linac and calculated dose from treatment planning system. The first one included using four different gamma criteria for local dose. These criteria are $3 \% / 3 \mathrm{~mm}, 3 \% / 5 \mathrm{~mm}, 4 \% / 4 \mathrm{~mm}$ and $5 \%$ dose difference (DD)/5 mm distance to agreement (DTA). It is performed by the suppression of the dose below either of $10 \%, 20 \%$ or $30 \%$ from the maximum of the reference matrix. The second test included using the same four gamma criteria but, in this case, for maximum dose. A comparison between local and maximum dose for every gamma criteria is then performed. 


\section{Results \& Discussion}

\section{Point dose measurements:}

The differences between the measured doses by ion chamber and that calculated for 30 patients (210 fields) using XIO TPS are expected to range from $-2.6 \%$ to $3.7 \%$ for whole plan (mean and standard deviation equal $0.46 \pm 1.7$ ) for head and neck tumors. In the home protocol one would expect the ionization chamber measurement to be within 3\% of calculated dose. If the result gives a variation higher than $5 \%$ the QA procedure is repeated. If the variations are between $3 \%$ and $5 \%$, the films or $2 \mathrm{D}$-array can be used for evaluation. The present results show that $96 \%$ of ion chamber measurements vary less than $5 \%$ from calculated dose and about $75 \%$ of ion chamber measurements vary less than $3 \%$ from calculated dose (Figure1). Dong et al. ${ }^{5}$ reported that the mean difference between measured and calculated doses was greater than $3.5 \%$. Chung et al. ${ }^{16}$ stated that the average difference between measured and computed dose at isocen- ter for a gantry angle $0^{\circ}$ for Head and Neck tumors was -0.55 \pm 1.51 . This corresponded to a range of variation of $-4.1 \%$ to $+3.9 \%$. Fenoglietto et al. ${ }^{17}$ reported a value of $1.33 \pm 3.22 \%$ for the difference between measured and calculated dose for head and neck tumors. Syam Kumar et al. ${ }^{15}$ reported also that a $0.6 \mathrm{~cm}^{3}$ ionization chamber gave $2.23 \%$ of the measured isocenter absolute dose which was comparable to the calculated plan. In the present results the differences may reach a maximum of $9 \%$ for an individual field. It has been cited that this difference might reach a maximum of $10 \% .{ }^{13}$ This large error most probably emerges from the variation in intensities of modulated beam resulting from the positioning of the ion chamber in the penumbra region of intensity map of the field. This may occur because of the size of farmer type ionization chamber or due to the charged particles equilibrium around the ion chamber (Figure 2). The same observation is reported by several authors. ${ }^{15}, 18,19,20$

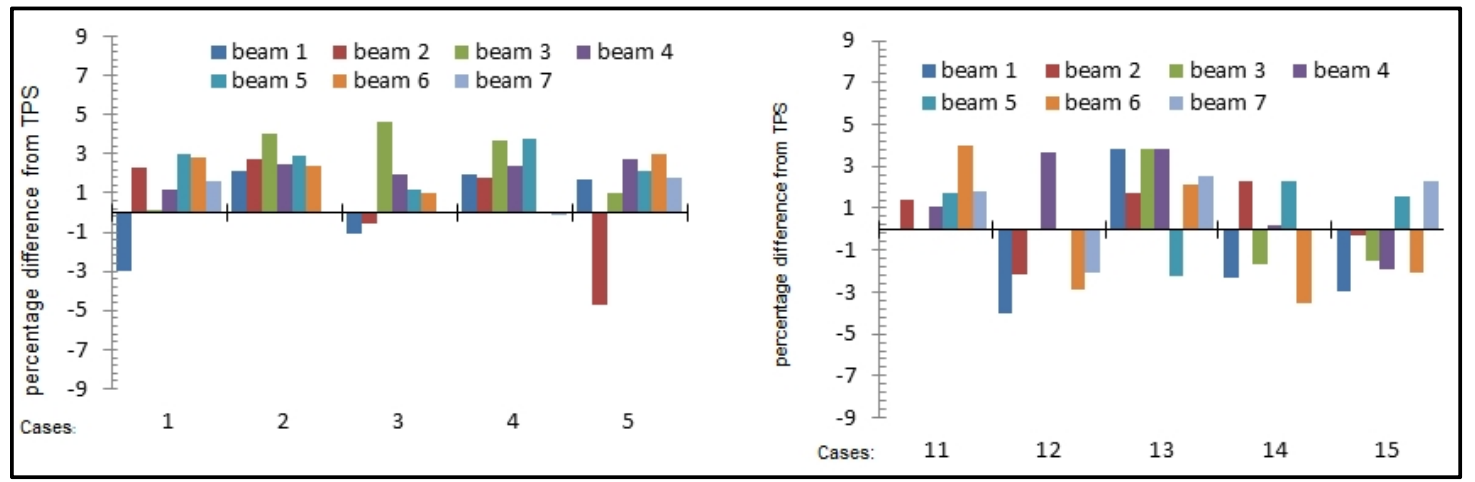

FIG. 1: Examples of relative difference in dose values between measured dose using $0.6 \mathrm{~cm}^{3}$ ionization chamber and calculated dose using XiO TPS.

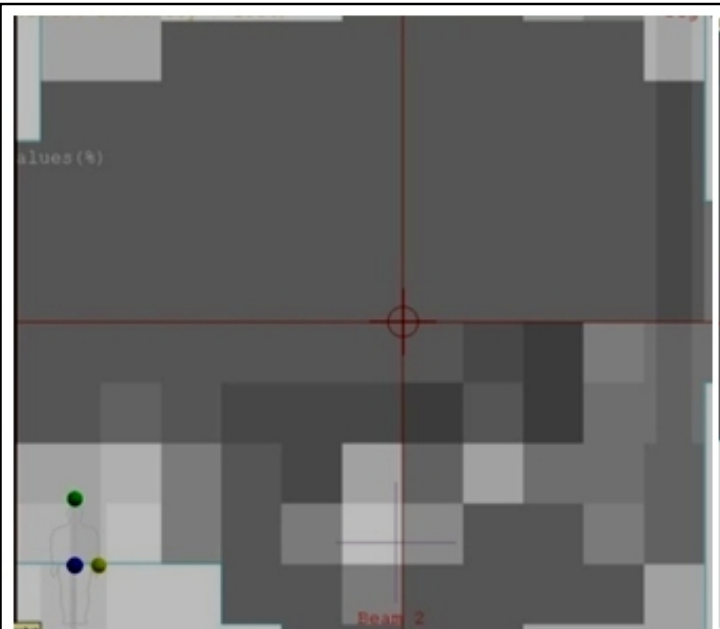

(a)

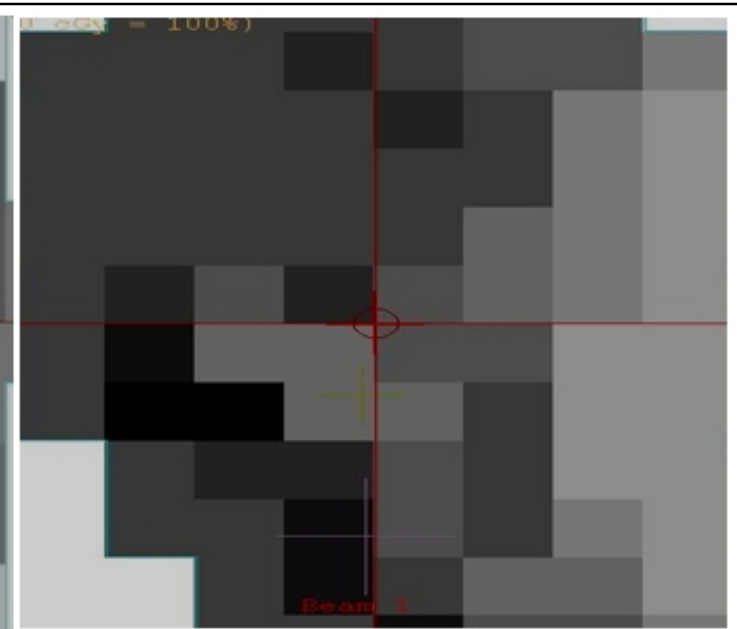

(b)

FIG. 2: Comparison between two fields having different intensities for head and neck IMRT cases: A large variation in intensity in the position of ion chamber can be seen. 


\section{Dose measurements using 2D-array}

Four different gamma criteria (dose difference(DD) /distance to agreement (DTA)) for 30 patients of Head and Neck tumors are compared to decide which criteria is to be used : $3 \% / 3 \mathrm{~mm}, 3 \% / 5 \mathrm{~mm}, 4 \% / 4 \mathrm{~mm}$ and $5 \% / 5 \mathrm{~mm}$ DD/DTA. Each of these criteria has been suggested elsewhere. ${ }^{2,}$, 21, 22 The choice of the criteria is made so that as much information as possible can be obtained and observed. The dose differences between measured dose (in Linac) and calculated dose (in TPS) are evaluated while suppressing the dose of $10 \%, 20 \%$ or $30 \%$ from dose distribution. A comparison between the results obtained for all gamma criteria is given below:

I. Local dose: The comparison between four different gamma criteria while suppressing the doses below $10 \%, 20 \%$ or $30 \%$ for 30 patients of head and neck tumors is performed. The criteria of $3 \% / 3 \mathrm{~mm}$ shows gamma passed $\%<90 \%$. On the other hand, the criteria of $5 \% / 5 \mathrm{~mm}$ shows gamma passed $\%$ greater than $95 \%$ for all suppression doses $(10 \%$,
$20 \%$, and $30 \%$ ). Figure 3 shows the gamma criteria of local dose excluding the lowest $20 \%$ of the dose distribution.

II. Maximum dose: the gamma passed \% dropped below $90 \%$ at $3 \% \mathrm{DD}, 3 \mathrm{~mm}$ DTA, nevertheless the average data for each criterion has gamma passed $\%$ values exceeding 90\%. Day et al. ${ }^{22}$ used the gamma criteria of $4 \%$ DD and $4 \mathrm{~mm}$ DTA with the lowest $10 \%$ of the dose distribution excluded from all gamma and DTA analyses for head and neck cases using 2D-array for the quality assurance (QA) of IMRT. Their result was $99.7 \%$ for the average gamma pass rate. While Zhang et al. ${ }^{23}$ found that the points passed ratio was more than $90 \%$ for 13\%/3 mm gamma criteria. The authors did not specify whether their published results were calculated for maximum dose or local dose. Figure 4 shows the gamma criteria of maximum dose excluding the lowest $20 \%$ of the dose distribution.

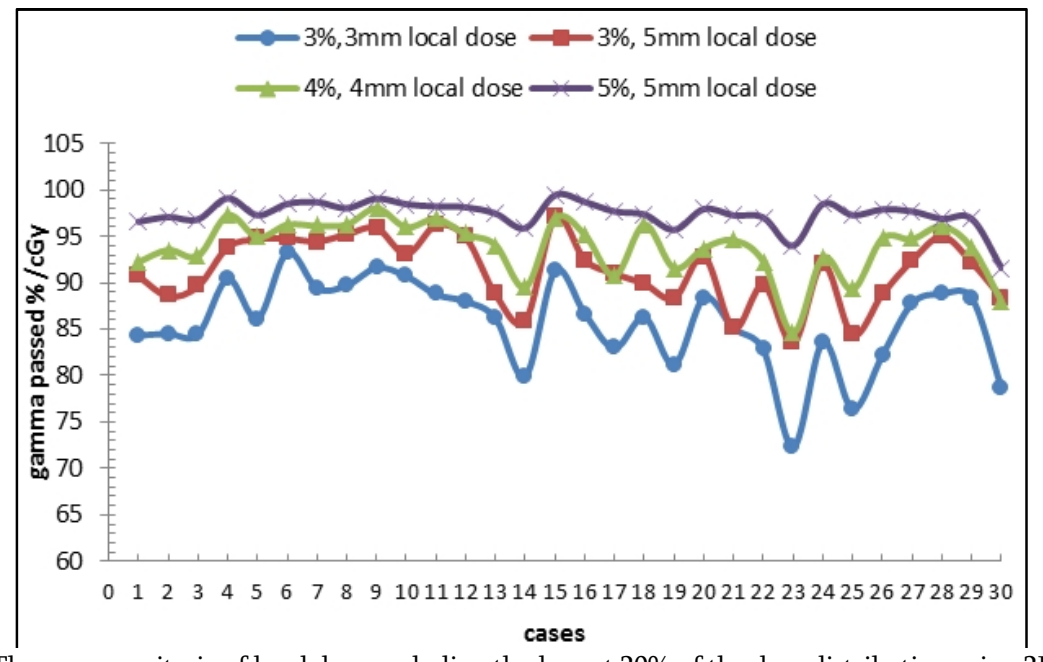

FIG. 3: The gamma criteria of local dose excluding the lowest $20 \%$ of the dose distribution using $2 \mathrm{D}$ array.

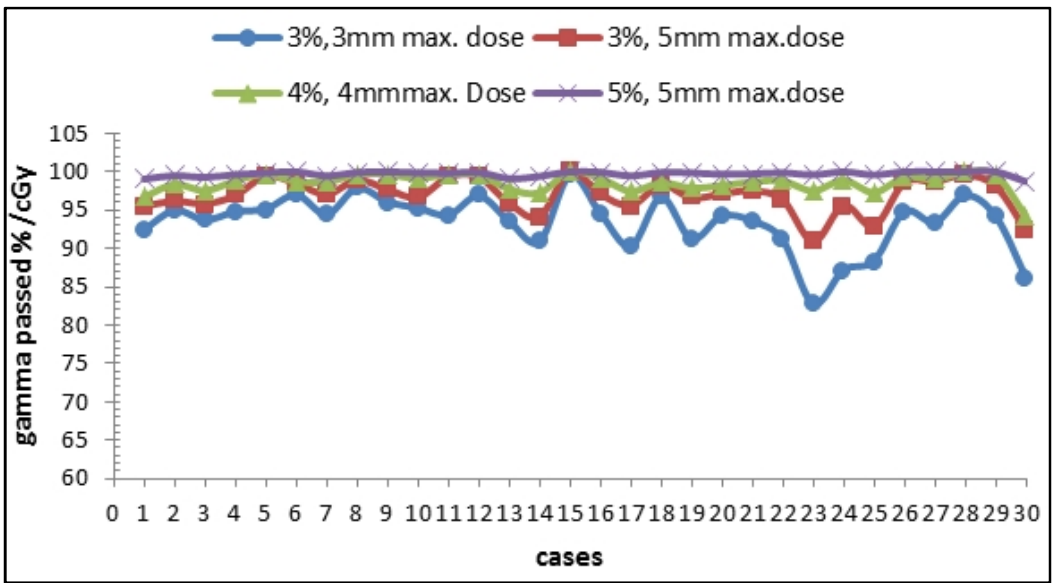

FIG. 4: The gamma criteria of maximum dose excluding the lowest $20 \%$ of the dose distribution using 2D array. 
A comparison between local and maximum doses for different gamma criteria:

I. When using gamma criteria of 3\% DD $3 \mathrm{~mm}$ DTA and suppressing dose below 10\%, the average and standard deviation for local dose of gamma index are $81.70 \pm 6.28$ while those for maximum dose of gamma index are $92.48 \pm 4.60$. In case of suppressing the dose below $20 \%$ the average and standard deviation for local dose are $85.67 \pm 4.74$ and for maximum dose are $93.42 \pm 3.64$. By increasing the value of suppressing dose below $30 \%$ the obtained data of average and standard deviation for local dose are $86.40 \pm 5.51$ and for maximum dose are $93.03 \pm 3.60$. From the above mentioned results, it is clear that the maximum dose data always show better results compared to local dose for all gamma values. This large reported difference between the gamma values obtained for maximum dose compared to local dose necessitates that different authors clarify whether their published results are calculated for maximum dose or local dose.

II. The calculations of average and standard deviation values for the other criteria ( $3 \% / 5 \mathrm{~mm}, 4 \% / 4 \mathrm{~mm}$ and $5 \% / 5 \mathrm{~mm})$ are listed in Table 1. All show greater gamma passed \% values for maximum dose compared to local dose.

TABLE 1: The average and standard deviation values for the all gamma criteria using 2D-array.

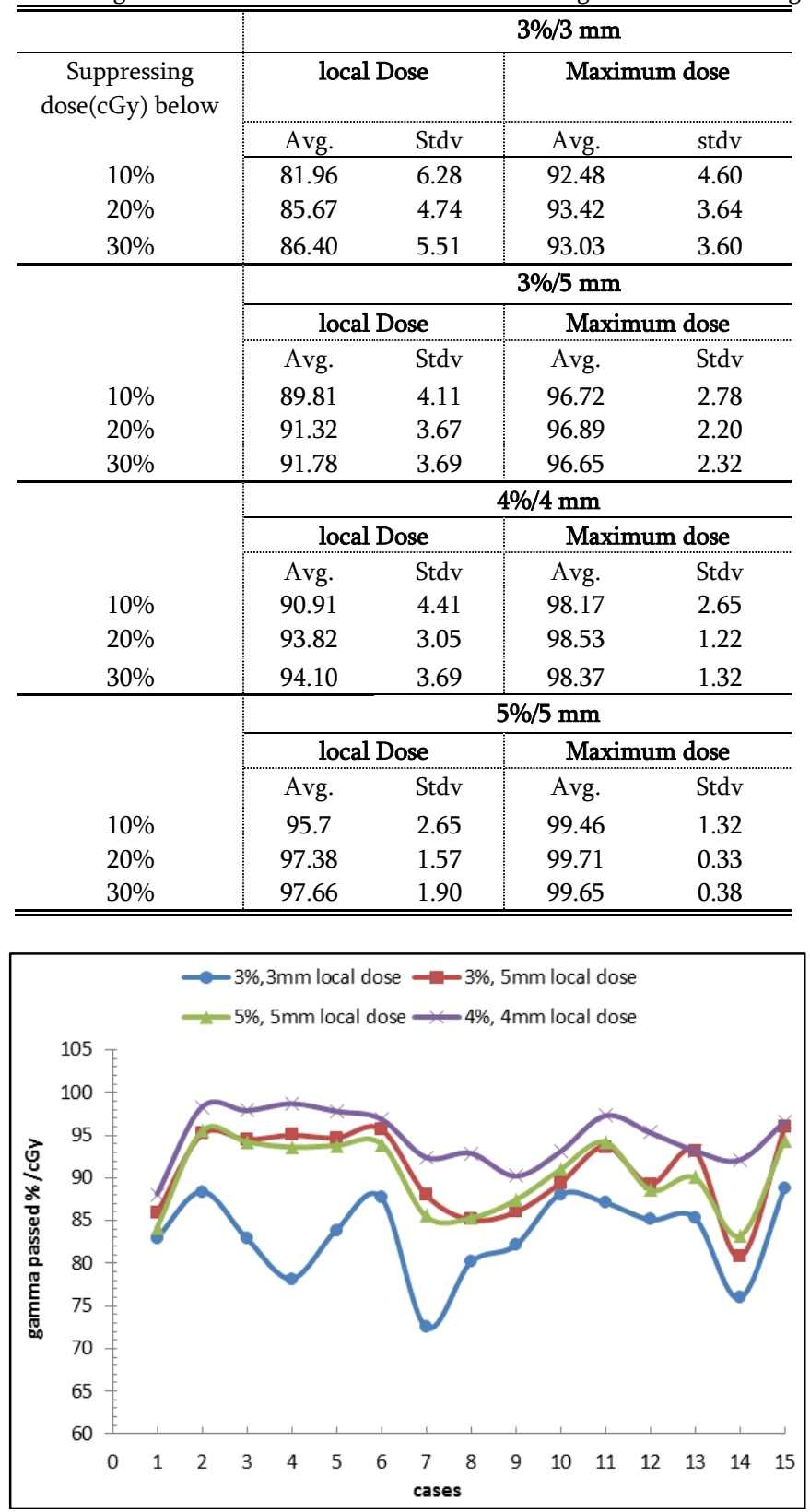

FIG. 5: The gamma criteria of local dose excluding the lowest $20 \%$ of the dose distribution using film. 


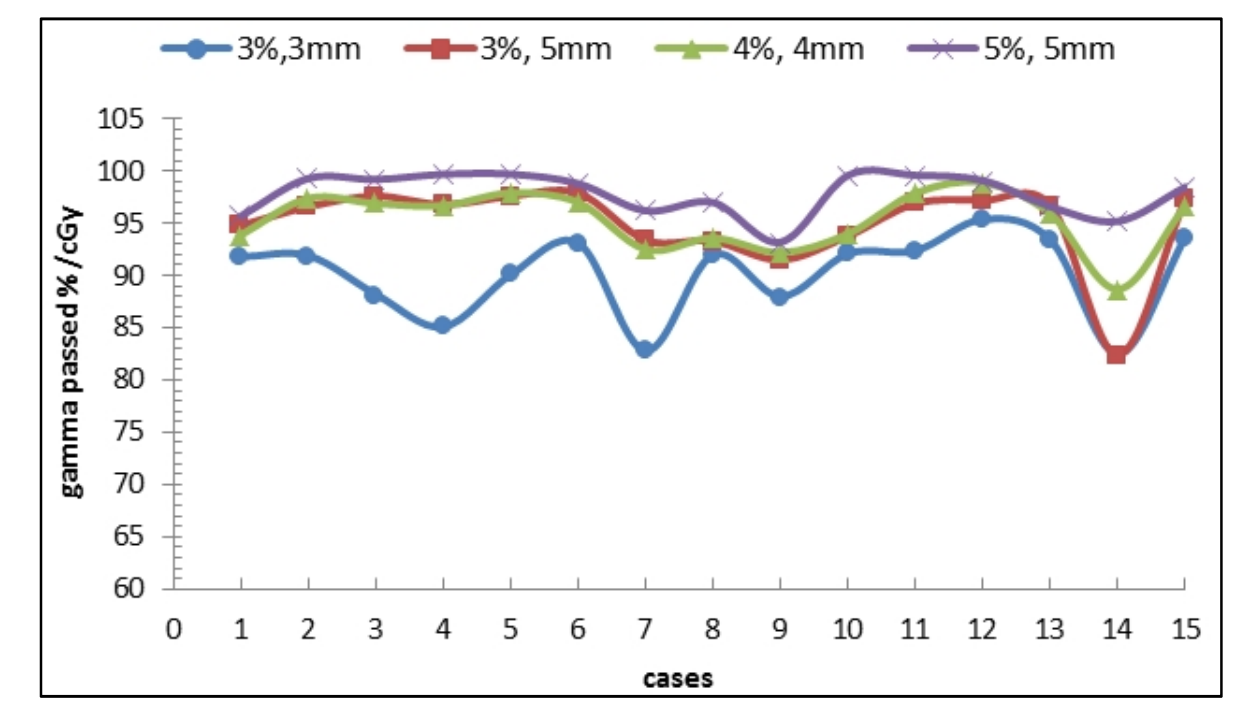

FIG. 6: The gamma criteria of maximum dose excluding the lowest $20 \%$ of the dose distribution using film.

\section{Dose measurements using Gafchromic EBT2 Films}

The same gamma criteria used in 2D-array measurements are applied for 15 head and neck cancer patients using Gafchromic EBT2 Films in order to compare efficiency of the different tools (2D-array vs. Gafchromic EBT2 Films). The obtained results are presented below:

I. Local dose: Similar to 2D-array, the data shows the highest gamma passed \% values (gamma passed \% $>90 \%$ ) for the $5 \%$ DD and $5 \mathrm{~mm}$ DTA gamma criteria while suppressing the dose below $30 \%$ for all gamma criteria. Also the criteria of $3 \% / 3 \mathrm{~mm}$ yield gamma values below $90 \%$ as in the case of 2D-array. The gamma criteria of local dose excluding the lowest $20 \%$ of the dose distribution using Film are shown in Figure 5.

II. Maximum dose: The acceptance limits for $y$ calculation are obtained for all gamma criteria. Unlike 2D array, the criteria of $3 \%$ DD and $3 \mathrm{~mm}$ DTA yield values of gamma passed \% less than 90\% when suppressing the dose below $10 \%$ only. All gamma criteria show highest values when suppressing the dose below 30\%. Anjum et al. ${ }^{2}$ compared between planned dose and irradiated dose distribution using GafChromic films. The gamma criteria of $3 \%$ dose difference (DD) and $3 \mathrm{~mm}$ distance to agreement (DTA) used for head and neck tumors in $96.3 \%$ of cases gave a gamma passed percent $\geq 75 \%$. The gamma criteria of maximum dose excluding the lowest $20 \%$ of the dose distribution using Film are shown in Figure 6.

The calculation of average and standard deviation values for all criteria $(3 \% / 3 \mathrm{~mm}, 3 \% / 5 \mathrm{~mm}, 4 \% / 4 \mathrm{~mm}$ and $5 \% / 5 \mathrm{~mm})$ are listed in Table 2 all show greater gamma passed \% values for maximum dose compared to local dose.

The results obtained with 2D-array are compared with numerous fluence distributions obtained with Gafchromic films for all criteria. Only minor differences in the value of gamma passed $\%$ are found. The data indicate a better performance for $2 \mathrm{D}$-array compared to film. The results are within $0.4 \%$ to $3.3 \%$ for local dose and $1.6 \%$ to $4.3 \%$ for maximum dose. This is an expected result due to the possible errors resulting from scanning, temperature, and the dose to optical density calibration of the film. Ahluwalia and Saini ${ }^{24}$ preferred the 2D-array than film and sates that the differences between them were within 3 to $5 \%$. Létourneau et al. ${ }^{25}$ also preferred the 2D-array than film because the use of 2D-array could reduce the IMRT QA workload. Saminathan et al. ${ }^{8}$ investigated the using of 2D-array in verification of IMRT comparing the data with films. The measurements and evaluation provided that 2D-array could be used for quantifying absolute dose and could also be used for routine quality assurance checks like flatness, symmetry, field width, and penumbra of the linear accelerator beam.

\section{Comparison between film and 2D-array \\ Local dose}

Statistical comparison between film and 2D-array for all criteria values while suppressing the $10 \%, 20 \%$ and $30 \%$ doses are carried out using Duncan multiple range test at $\mathrm{p}<$ 0.01.Significant differences are observed between the films and $2 \mathrm{D}$-array only for $3 \% / 3 \mathrm{~mm}$ criteria (for $10 \%$ dose suppression), $4 \% / 4 \mathrm{~mm}$ and $5 \% / 5 \mathrm{~mm}$ criteria (for $10 \%$ and $20 \%$ dose suppression). 
TABLE 2: The average and standard deviation values for the all gamma criteria using film.

\begin{tabular}{|c|c|c|c|c|}
\hline & \multicolumn{4}{|c|}{$3 \% / 3 \mathrm{~mm}$} \\
\hline \multirow{2}{*}{$\begin{array}{c}\text { Suppressing } \\
\text { dose(cGy) below }\end{array}$} & \multicolumn{2}{|c|}{ local Dose } & \multicolumn{2}{|c|}{ Maximum dose } \\
\hline & Avg. & Stdv & Avg. & stdv \\
\hline $10 \%$ & 80.19 & 4.73 & 88.45 & 4.04 \\
\hline $20 \%$ & 83.27 & 4.69 & 90.17 & 3.86 \\
\hline \multirow{4}{*}{$30 \%$} & 84.51 & 6.07 & 90.57 & 4.98 \\
\hline & \multicolumn{4}{|c|}{$3 \% / 5 \mathrm{~mm}$} \\
\hline & \multicolumn{2}{|c|}{ local Dose } & \multicolumn{2}{|c|}{ Maximum dose } \\
\hline & Avg. & Stdv & Avg. & Stdv \\
\hline $10 \%$ & 88.74 & 3.70 & 93.88 & 3.56 \\
\hline $20 \%$ & 90.92 & 4.40 & 94.81 & 4.23 \\
\hline \multirow[t]{4}{*}{$30 \%$} & 91.63 & 5.05 & 94.98 & 4.66 \\
\hline & \multicolumn{4}{|c|}{$4 \% / 4 \mathrm{~mm}$} \\
\hline & \multicolumn{2}{|c|}{ local Dose } & \multicolumn{2}{|c|}{ Maximum dose } \\
\hline & Avg. & Stdv & Avg. & Stdv \\
\hline $10 \%$ & 88.66 & 4.24 & 94.29 & 3.28 \\
\hline $20 \%$ & 90.69 & 3.73 & 94.98 & 3.74 \\
\hline \multirow[t]{4}{*}{$30 \%$} & 91.51 & 4.32 & 95.13 & 4.19 \\
\hline & \multicolumn{4}{|c|}{$5 \% / 5 \mathrm{~mm}$} \\
\hline & \multicolumn{2}{|c|}{$\begin{array}{l}\text { local Dose } \\
\text { locan }\end{array}$} & \multicolumn{2}{|c|}{ Maximum dose } \\
\hline & Avg. & Stdv & Avg. & Stdv \\
\hline $10 \%$ & 92.54 & 3.73 & 97.23 & 1.79 \\
\hline $20 \%$ & 94.72 & 3.14 & 97.83 & 1.97 \\
\hline $30 \%$ & 95.33 & 3.47 & 98.03 & 2.21 \\
\hline
\end{tabular}

\section{Maximum dose}

Statistical comparison between film and 2D-array for all criteria values while suppressing the $10 \%, 20 \%$, and $30 \%$ doses are carried out using Duncan multiple range test at $\mathrm{p}<$ 0.01.Significant differences are observed between the films and $2 \mathrm{D}$-array for all criteria except the $3 \% / 5 \mathrm{~mm}$ criteria.

\section{Conclusion}

It is clear that the maximum dose data always show better results compared to local dose for all gamma values which are performed by films and 2D-array. The films and 2D-array for local dose show best values for $5 \% \mathrm{DD}$ and 5 mm DTA for gamma passed $\%>90 \%$. Also the best values are obtained when suppressing the dose below 30\% for all gamma criteria. The criteria of $3 \% / 3 \mathrm{~mm}$ show gamma values below $90 \%$. For maximum dose when using the film, the criteria of $3 \% \mathrm{DD}$ and $3 \mathrm{~mm}$ DTA give values of gamma passed \% less than $90 \%$ when suppressing the dose below $10 \%$ only. All gamma criteria produced the highest values when suppressing the dose below $30 \%$. Unlike film when using $2 \mathrm{D}$-array, the average data for each criterion has gamma passed \% exceeding 90\%.This large reported difference between the gamma values obtained for maximum dose compared to local dose necessitates that different authors clarify whether their published results are calculated for maximum dose gamma index or local dose gamma index.

The data indicate also a better performance for 2D-array compared to film. The results obtained with $2 \mathrm{D}$-array are compared with numerous fluence distributions obtained with Gafchromic films for all criteria. Only minor differences in the value of gamma passed \% are reported. These differences are within $0.4 \%$ to $3.3 \%$ for local dose and $1.6 \%$ to $4.3 \%$ for maximum dose. This is an expected result due to the possible errors resulting from scanning, temperature and the dose to optical density calibration of the film. The use of 2D-array can reduce the IMRT QA workload. Based on the results of this work, the criteria of $3 \% \mathrm{DD}$ and $5 \mathrm{~mm}$ DTA while suppressing the dose below $20 \%$ are recommended. These criteria are also reported by Anjum et al. ${ }^{2}$. Under this criterion the percent of pixels passing gamma is $>95 \%$ in case of maximum dose.

\section{Conflict of interest}

The authors declare that they have no conflicts of interest. The authors alone are responsible for the content and writing of the paper. 


\section{References}

1. Ezzel G A, Galvin J M, Low D, et al. Guidance document on delivery, treatment planning, and clinical implementation of IMRT: report of the IMRT Subcommittee of the AAPM Radiation Therapy Committee. MedPhys 2003; 30:2089 -115.

2. Anjum MN1, Parker W, Ruo R, Afzal M. Evaluation criteria for film based intensity modulated radiation therapy quality assurance. Phys Med 2010; 26:38-43.

3. Burman C, Chui CS, Kutcher G, et al. Planning, delivery, and quality assurance of intensity-modulated radiotherapy using dynamic multileaf collimator: a strategy for large-scale implementation for the treatment of carcinoma of the prostate. Int J Radiat Oncol Biol Phys 1997; 39: 863-73.

4. Kron T, Hamilton C, Roff M, Denham J. Dosimetric intercomparison for two Australasian clinical trials using an anthropomorphic phantom. Int $J$ Radiat Oncol Biol Phys 2002; 52:566-79.

5. Dong L, Antolak J, Salehpour M, et al. Patient-specific point dose measurement for IMRT monitor unit verification. Int J Radiat Oncol Biol Phys 2003; 56: 867-77

6. Low DA and Dempsey JF. Evaluation of the gamma dose distribution comparison method. Med Phys 2003; 30:2455-64.

7. Childress NL, White RA, Bloch C, et al. Retrospective analysis of 2D patient-specific IMRT verifications. Med Phys 2005; 32:838-50.

8. Saminathan S, Manickam R, Chandraraj V, Supe S. Dosimetric study of 2D ion chamber array matrix for the modern radiotherapy treatment verification. J Appl Clin Med Phys 2010; 11:3076.

9. Eisbruch A, Ship JA, Martel MK, et al. Parotid gland sparing in patients undergoing bilateral head and neck irradiation: techniques and early results. Int J Radiat Oncol Biol Phys 1996; 36:469-80.

10. Ozyigit G, Yang T, Chao KS. Intensity-modulated radiation therapy for head and neck cancer. Curr Treat Options Oncol 2004; 5:3- 9.

11. ICRU Report 83, Prescribing, Recording, and Reporting Photon-Beam Intensity-Modulated Radiation Therapy (IMRT). Journal of the ICRU 2010; 10.

12. Low DA, Moran JM, Dempsey JF, et al. Dosimetry tools and techniques for IMRT. Med Phys 2011; 38: 1313-38.

13. Mijheer B, Georg D. Guidelines for the verification of IMRT. ESTRO BOOKLET NO. 9: Brussels, Belgium, 2008.

14. Agnew CE, Irvine DM, Hounsell AR, McGarry CK. Improvement in clinical step and shoot inten- sity modulated radiation therapy delivery accuracy on an integrated linear accelerator control system. Pract Radiat Oncol 2014; 4:43-9.

15. Syam Kumar SA, Sukumar P, Sriram P, et al. A patient-specific quality assurance study on absolute dose verification using ionization chambers of different volumes in RapidArc treatments. Med Dosim 2012; 37: 436-41.

16. Chung H, Li J, Samant S. Feasibility of using two-dimensional array dosimeter for in vivo dose reconstruction via transit dosimetry. J Appl Clin Med Phys 2011; 12:3370.

17. Fenoglietto $P$, Laliberté $B$, Aillères $N$, et al. Eight years of IMRT quality assurance with ionization chambers and film dosimetry: experience of the Montpellier Comprehensive Cancer Center. Radiat Oncol 2011; 6:85.

18. Escudé L, Linero D, Mollà M, Maralbell R. Quality assurance for radiotherapy in prostate cancer: point dose measurements in intensity modulated fields with large dose gradients. Int JRadiat Oncol Biol Phys 2006; 66: S136-40.

19. Laub WU and Wong T. The volume effect of detectors in the dosimetry of small fields used in IMRT. Med Phys 2003; 30:341-347.

20. Sánchez-Doblado F, Hartmann GH, Pena J, et al. Uncertainty estimation in IMRT absolute dosimetry verification. Int J Radiat Oncol Biol Phys 2007; 68: 301-10.

21. Li Q Li L, Han J, Liang Z. Dose distribution verifications of IMRT for NPC. J Huazhong Univ Sci Technolog Med Sci 2009; 29:673-6.

22. Day RA, Sankar AP, Nailon WH, MacLeod AS. On the use of computed radiography plates for quality assurance of intensity modulated radiation therapy dose distributions. Med Phys 2011; 38:632-45

23. Zhang Y, Gao Y. Dosimetric verification of the intensity modulated radiation therapy. Zhongguo $Y i$ Liao Qi Xie Za Zhi 2010; 34:218-20, 223.

24. Ahluwalia B, Saini DS. IMRT QA with 2-dimensional Diode Array of Detectors. Presented at ICMP and Silver Jubilee AMPI Conference, New Delhi, India October 2004. Available from: http://sunnuclear.com/Papers/MapCHECKPaper.pd f

25. Létourneau D, Gulam M, Yan D, et al. Evaluation of a 2D diode array for IMRT quality assurance. Radiother Oncol 2004; 70:199-206. 